\title{
Un estudio de Género con Enfoque Territorial: La participación femenina en pequeñas comunidades rurales de Brasil y Uruguay
}

\author{
A S tudy of Gender with a Territorial Approach: Women's participation in small rural \\ communities in Brazil and Uruguay
}

\author{
Rossana Vitelli \\ Universidad de la República \\ vitellirossana@gmail.com
}

Resumen

En este artículo se presentan algunos de los resultados encontrados en una investigación realizada en Brasil y Uruguay, donde se intenta demostrar cómo la participación -especializada $\mathrm{y}$ diferenciada por género- de las mujeres en redes y organizaciones del medio rural, produce capital social y ayuda a los procesos de fortalecimiento de la ciudadanía; siendo que el territorio marca en forma visible algunas distinciones de la forma en que se dibujan estos procesos. En la primera parte se comparten algunos elementos conceptuales que integran el debate sobre género, capital social, participación y territorio. En el segundo capítulo se presentan datos de la investigación realizada durante los años 2008 y 2009 en las comunidades de San Pedro (Colonia) en Uruguay y Santa Cruz do Sul en Brasil.

Palabras-clave: mujeres rurales; desarrollo; capital social.

\begin{abstract}
This article presents some results produced by a research accomplished both in Brazil and Uruguay. It tries to show how women's participation in networks and rural organizations produces social capital and enforces the process of making citizenship stronger. It is pretty clear that the territory makes distinctions in the way how this participation and social capital is produced. In the first chapter, we introduce some concepts that are important for the debate on gender equity, participation and territory. In the second chapter, we present some data gathered from the research made during the years 2008 and 2009, in the communities of San Pedro (Colonia), in Uruguay, and Santa Cruz do Sul, in Brazil.
\end{abstract}

Keywords: rural women; development; social capital. 


\section{Introducción}

En este trabajo se presentan algunos resultados obtenidos a partir de una investigación más amplia realizada en San Pedro, departamento de Colonia en Uruguay y Santa Cruz do Sul, Estado de Rio Grande do Sul en Brasil. Se intenta realizar un aporte en el sentido de presentar el debate de algunos conceptos teóricos que se reconocen polisémicos y complejos como lo son el de capital social, el de participación y la misma concepción de ciudadanía, a partir de los cuales se hizo el esfuerzo de construir categorías e indicadores que pudieran hacerlos observables empíricamente.

La vitalidad y desarrollo de las comunidades rurales están construidos sobre los pilares de un conjunto de organizaciones, grupos y asociaciones de diverso tipo que a través de su accionar generan un conjunto de recursos y bienes que pueden ser considerados como capital social. La participación de las mujeres rurales en este tipo de vida comunitaria no había sido suficientemente estudiada y con frecuencia poco valorada y reconocida. Los cuadros que se presentan a continuación son parte de la encuesta realizada, y miden sólo una de las dimensiones de las varias utilizadas en su momento. Seleccionamos el tema de la participación en grupos y organizaciones.

\section{El Desarrollo Rural Sustentable, la Participación en Redes y el Capital Social}

El aporte que las mujeres realizan al desarrollo en el medio rural y sus comunidades a través de su participación en organizaciones sociales y los roles que cumplen, además de ser cualitativamente distinto al de los hombres, es poco reconocido en términos de la 'normalización' que se ha dado de esas funciones y de la poca jerarquía dada a estas actividades.

Se consideró para esta investigación el conjunto de tareas y funciones sociales que realizan las mujeres en sus localidades, en términos de su participación organizada en instituciones, y también otras actividades que conforman un conjunto más inorgánico de redes comunitarias y solidarias de la comunidad. Estas formas de participación se consideran generadoras de capital social tanto para las propias mujeres como para la comunidad toda, así como mecanismos de ampliación de las prácticas de ciudadanía.

\section{La Participación como Elemento Motor}

El primer elemento que nos interesa definir es la participación. Este es un concepto complejo que a efectos de la investigación realizada, ha sido de utilidad como forma de posibilitar una observación de forma más empírica los mecanismos que construyen capital social y espacios de ciudadanía.

Si bien el concepto de 'participar' podría considerarse neutro, ni bueno ni malo en sí mismo, para algunos autores la participación produce influencias positivas no sólo a nivel del cuerpo social sino también del individuo. En este enfoque, la no participación es posible fuente de desequilibrios como afirma Mitscherlich (DEL ACEBO IBAÑEZ, 2006).

Es posible entender la participación en dos formas de manifestarse: activa y pasiva. Esta última acepción se refiere a la posibilidad de acceso a ciertos bienes y servicios públicos que generalmente son brindados a través de políticas públicas. Por otro lado la participación activa es la que adquiere el sujeto a través de su protagonismo en un sistema más general de toma de decisiones a distinto nivel. De acuerdo con F.Tönnies esta participación activa puede adoptar formas comunitarias o societarias cuando el individuo intenta modificar la realidad en la que vive. (DEL ACEBO IBAÑEZ,E; op.cit.)

La participación comunitaria adquiere una importancia y significación propia cuando se da a nivel vecinal o barrial, ya que la misma permite cierto anclaje del individuo a su localidad. Este accionar le otorga al sujeto un sentimiento de pertenencia al lugar y de identificación al poder compartir con 'iguales' que son sus vecinos ciertas instancias. Estos componentes subjetivos de identificación al integrarse a un grupo de iguales pueden convertirse en uno de los primeros motivadores que hacen que las personas participen.

En síntesis, se considerará la participación como el motor que moviliza en primer lugar a las personas para integrarse a distintos tipos de grupos $y$ formas asociativas, siendo éste el primer paso necesario para que se den los otros dos componentes: el de la generación de capital social y el de la producción de ciudadanía. El hecho que finalmente se generen o no estos últimos, dependerá del conjunto total de contenidos que tanto las organizaciones como los sujetos logren imprimir y reproducir en sus acciones. Este conjunto de contenidos que llamaremos 'dispositivo normativo', está integrado por valores, tradición, religión e ideología y a su vez por consideraciones éticas y culturales. 


\section{Ciudadanía, Capital Social y Mujeres en el Enfoque Territorial}

Existe un espacio teórico donde territorio, capital social y ciudadanía se articulan. Es un punto donde se asocian estos conceptos para poder discutir un modelo de desarrollo equilibrado, sustentable y equitativo. Tal como lo consideramos en este trabajo, el territorio es un recurso social, colectivo e indivisible. De esta forma, los modos de gestión y administración del mismo suponen necesariamente formas de organización para un desarrollo de la toma de decisiones y gerenciamiento de ese espacio colectivo.

Las formas, los medios, y todos los mecanismos que la sociedad local se da a sí misma para estos fines de administración y gestión del territorio, están inspiradas en las concepciones históricas e ideológicas, valorativas y culturales que sus habitantes tengan $\mathrm{y}$ hayan construido con respecto a las concepciones sobre ciudadanía, participación y el involucramiento en lo social. Desde una perspectiva político-liberal podrá sustentarse la necesaria 'libertad' de los agentes privados individuales para actuar y negociar con un Estado a través de su administración centralizada; desde teorías más reformistas cercanas a las del 'welfare' se puede justificar la organización colectiva de los sujetos para asumir en forma más descentralizada y más participativa. Por tanto, estos mecanismos que las sociedades construyen para organizar sus espacios, son pasibles de ser examinados en términos de 'construcción de ciudadanía' cuando permiten, pero sobre todo cuando 'transmiten' formas colectivas de gestión de su territorio. Esto indica una valoración positiva acerca de la participación, así como conocimiento y transmisión de los derechos ciudadanos.

La necesidad de administrar un bien colectivo como el territorio, igualmente implica la cooperación y la asociación de los sujetos, lo que genera a la vez, gestión del espacio y capital social. Los distintos tipos de capital social que se dan: individual o colectivo, horizontal o vertical, también están altamente asociados a la cultura cívica y a los valores que cada territorio imprime para sí. El territorio tiene una dimensión histórica importante en donde desde distintas miradas han quedado grabados un patrimonio ambiental, valorativo, simbólico y también material. Por tanto los bienes producidos como capital social, forman parte de ese acervo local y territorial.

También forma parte de ese patrimonio un componente identitario y de pertenencia muy fuerte, que imprime 'lo local' a las formas en que los sujetos se 'sienten' ciudadanos y a los mecanismos en que éstos construyen para la generación del capital social.
La articulación entre ambos conceptos es observable si se entiende que 'La producción de representación, valores y códigos compartidos, la socialización de las experiencias, no solamente genera confianza entre los actores: también contribuye a la construcción de su identidad social" (Riella, 2005) Estamos afirmando que a la vez que se genera capital social, se conforma la identidad del territorio.

\section{Capital Social y Desarrollo en el Medio Rural}

Para este estudio se recogieron algunos aspectos esenciales de las teorías desarrolladas por Bourdieu, Putnam y Coleman, en el contexto de que si bien parten de enfoques conceptuales diferentes, existen elementos que se complementan y no necesariamente entran en contradicción. En síntesis podríamos afirmar que si Bourdieu -para explicar el capital socialemplea aspectos de tipo estructurales y económicos, Coleman enfatiza los rasgos más subjetivos y características de los individuos -como ser la confianza y la cooperación- mientras que Putnam apela más a una perspectiva sociocultural y a los procesos históricos del civismo. Se intentó realizar una síntesis de estas teorías utilizando algunos de los elementos que fueran de mayor utilidad para el estudio. De acuerdo con lo afirmado por algunos trabajos de la CEPAL, 'pese a la polisemia del concepto, hay ciertos elementos comunes...' (ARRIAGADA, 2003). Hay que precisar que, en virtud de trasformar estos conceptos en una herramienta de utilidad analítica, se recogieron algunos trabajos posteriores que intentan superar algunas críticas realizadas sobre las primeras formulaciones del capital social, y que se enfatizaron sus aspectos más susceptibles de articularse con los de participación y ciudadanía.

En este caso, estamos comprendiendo al capital social como un conjunto de recursos disponibles en la estructura de la sociedad, y de los cuales los sujetos pueden disponer, a través de sus redes e instituciones y que están necesariamente imbricadas con su sistema de normas y valores. De este modo se coincide con Arriagada cuando afirma que el capital social de un grupo podría entenderse como "la capacidad efectiva de movilizar, productivamente $\mathrm{y}$ en beneficio del conjunto, los recursos asociativos que radican en las distintas redes sociales a las que tienen acceso los miembros del grupo". (ARRIAGADA, op.cit.). Para John Durston -quien ha desarrollado esta teoría aplicada al medio rural- el capital social descansa en las relaciones de confianza y cooperación que se operan en base a un criterio de reciprocidad 'difusa', 
Durston (2002).

Desde nuestro enfoque, la utilidad de esta teoría aplicada al medio rural es la de ayudar a explicar y de estar íntimamente vinculada a los aspectos del desarrollo y el crecimiento de las pequeñas comunidades. Si seguimos a Granovetter (1985) y su concepto de 'embededness' (entendido como 'imbricamiento') de las relaciones económicas con las sociales; se puede considerar de importancia examinar cómo en el medio rural las estrategias de vida, así como los mecanismos de crecimiento económico y las actividades productivas de las comunidades están fuertemente vinculadas y dependen en muchos casos de las formas de capital social existentes de una manera diferente que en las ciudades, donde la disposición de recursos y servicios pueden sustituirlas. Igualmente estas formas de capital social pueden ayudar a explicar las desigualdades en sus procesos de desarrollo en territorios que cuenten con similares condiciones de partida. Por estas razones también se puede compartir con Woolcock cuando afirma que:

la pobreza en sentido estrictamente económico puede ser tan intensa en el campo como en la ciudad, pero la presencia en el campo de redes comunitarias más estrechas y duraderas impide muchas de las peores manifestaciones de la pobreza urbana, Woolcock (1998)

\section{Capital Social y Procesos de Ciudadanía Femenina}

Los aspectos que vinculan al capital social con la ciudadanía fueron con claridad expresados por Cristobal García cuando afirma que:

La Ciudadanía nos introduce en el debate propio de la teoría política, mientras que el Capital Social y la Solidaridad nos permite el enlace con la experiencia de los vínculos y prácticas sociales que constituyen, en buena medida, el corpus sobre el que se construyen las teorías del orden sociopolítico (CRISTÓBAL GARCÍA H., 2001)

El interés teórico del problema, radica en la discusión de los mecanismos que permiten que la participación de las mujeres rurales en redes informales, y en instituciones y organizaciones de la comunidad, terminen siendo formas de ejercicio de ampliación de la ciudadanía no sólo para ellas, sino para el conjunto de la comunidad que se beneficia de estos procesos, colaborando de ese modo en los procesos de desarrollo.

Estamos concibiendo la idea de ciudadanía como 'un constructo siempre en movimiento'. Hopenhayn señala las transformaciones en los derechos al "reinscribirse la ciudadanía en nuevos espacios, mostrando que efectivamente 'la ciudadanía es un concepto y una práctica en mutación' (AGUIRRE, 2003). Es, entonces, un proceso histórico, una construcción social y que por tanto debe redefinirse continuamente. Podemos acordar acá con Coutinho en una forma de entender la ciudadanía como:

capacidad conquistada por algunos individuos o (en caso de una democracia efectiva) por todos los individuos, de apropiarse de los bienes socialmente creados, de actualizar todas las potencialidades de realización humana abiertas por la vida social en cada contexto históricamente determinado. (COUTINHO,1999) ${ }^{1}$

Para este autor los individuos no nacen con derechos, (como en el jusnaturalismo) sino que los derechos son fenómenos sociales, y por tanto resultado de la historia. Así es que siempre son logros obtenidos por las demandas realizadas por distintos grupos sociales a través de sus luchas y movilizaciones.

De hecho, parece importante destacar la dimensión contextual del debate acerca de la ciudadanía, y la tensión existente entre la ciudadanía 'formal' y la 'sustantiva'. La discusión sobre los derechos sociales de la ciudadanía debería considerar que los mismos deben adquirir cierta autonomía e independencia del lugar de origen de los sujetos y ser válidos o universales en el lugar que trabaja o vive. Sin embargo los procesos de globalización que suponen por períodos grandes contingentes migratorios tanto legales como ilegales, centran permanentemente el dilema en cuestiones de 'nacionalidad'.

En este marco es que se inserta el debate sobre la cuestión de la ciudadanía ejercida por las mujeres. Es decir, un complejo entramado donde las demandas por el reconocimiento de los derechos de las mujeres se articulan junto con las demandas realizadas por otros grupos sociales para ser reconocidos: por su condición étnica, religiosa, orientación sexual, etc., por entender que su acceso al ejercicio pleno de sus derechos está limitada por su pertenencia a una minoría. En muchos países las mujeres tienen poco poder de decisión sobre su propia salud y cuerpo, o tienen sus derechos civiles y políticos restringidos. Numerosas investigadoras explican que existe una 'debilidad de origen' en el 
Un estudio de Género con Enfoque Territorial: La participación femenina en pequeñas comunidades rurales de Brasil y Uruguay

concepto de ciudadanía, desde que en su nacimiento bajo la concepción liberal, el portador de la ciudadanía era el hombre, y la mujer era esposa y madre de los ciudadanos. La incorporación de los beneficios de la ciudadanía social ha tenido impactos diferentes en hombres y mujeres. (AGUIRRE, op.cit.) Esto es sencillo de entender si se considera que el Estado de Bienestar que implementó las políticas de atención a la salud, jubilaciones, desempleo, tomó en cuenta al trabajador asalariado y a la familia como una unidad, por lo reprodujo las inequidades de género preexistentes.

En este trabajo nos centramos en aquellos mecanismos de generación de capital social que -a través de formas organizadas de cooperación, gestión, y participación- permiten que se formen espacios para la ampliación de la práctica y el ejercicio de la ciudadanía femenina, ya que en esos espacios se pueden encontrar modos genuinos de producción, redistribución, y apropiación de recursos y bienes sociales que hacen al desarrollo comunitario. En este sentido, se piensa el desarrollo como: equitativo, sustentable y equilibrado.

\section{Las Caracteristicas de la Participacion Femenina en San Pedro y Santa Cruz do Sul}

\section{Metodología y Técnicas Utilizadas}

Para realizar esta investigación se plantearon una serie de actividades en dos comunidades: Santa Cruz do Sul, Estado de Rio Grande do Sul en Brasil, y San Pedro, Departamento de Colonia en Uruguay. La selección de dos localidades en dos países, tuvo como sustento argumentativo y teórico el poder ampliar el universo e incorporar al estudio la incidencia de factores, tanto tradicionales como culturales, de cada país. No se trata en sentido estricto de un estudio comparado, si bien se eligieron comunidades con rasgos de comparabilidad suficiente como para ser analizados en forma conjunta.

Si bien el abordaje metodológico general utilizado fue el cualitativo, entendiendo que era el más adecuado para el objeto de estudio, también se realizó una encuesta, a los efectos de recabar la información necesaria y poder construir un 'cuerpo de datos' consistente y lograr una triangulación de técnicas. A los efectos de este artículo se seleccionó parte de los resultados de la encuesta que fuera realizada con las siguientes características:
- Se trató de una encuesta probabilística y de tipo aleatoria, con una muestra representativa para la población local-rural a los vecinos de Santa Cruz y San Pedro. Se encuestaron personas mayores de 18 años respetando la proporción de población y género para cada localidad.

\section{Algunos de los Hallazgos Encontrados}

La participación activa de los vecinos en acciones y organizaciones que trabajan para la comunidad no es relevante como práctica que se agota en sí misma; sino que interesa analizarla en un marco más amplio que incluya sus motivaciones, los objetivos que se plantea, los impactos y movilizaciones que produce; y -finalmente- las transformaciones que promueve tanto en lo colectivo como en el propio individuo. En este sentido se consideró 'participación' en su concepción más amplia, como toda aquella actividad que relacione y acerque a los vecinos en tanto pueda cumplir varias funciones tanto individuales como colectivas. En otro momento de la investigación se analizó en qué medida esas formas de agrupamiento tenían eventualmente la posibilidad de generar ciudadanía. Ese aspecto no lo incorporamos en este punto por exceder los cometidos de la presentación. Se efectuaron una serie de preguntas para indagar los distintos tipos y grados de participación de los vecinos en la comunidad. Sobre este tema nos interesó tanto la propia práctica de participación del encuestado como sus percepciones acerca del resto de la comunidad.

La primera de las preguntas sólo consultaba si el encuestado participaba o formaba parte de alguna organización o grupo.(Grafico 1)

El porcentaje de personas que son parte de algún colectivo, sea con mayor o menor grado de formalidad es -sin lugar a dudas- francamente elevado representando un $66 \%$ de los entrevistados. Es cierto que en esta primer pregunta, se registra en forma muy genérica cualquier y todo tipo de grupo u organización, lo que -como se verá después- significa una heterogeneidad muy grande. Este, sin embargo, es el primer indicador que muestra los grados en que los vecinos tienen grupos de referencia y/o pertenencia, lo que ya de por sí es relevante.

Examinando las respuestas por comunidad, existe una diferencia significativa a favor de San Pedro, donde los que participan en algún grupo son el $83 \%$. Esta información da elementos muy fuertes para diagnosticar esta comunidad. Esta altísima tasa indica que los lazos y el entramado de vínculos entre los vecinos son muy fuertes. En Santa Cruz do Sul -igualmente- es casi la mitad de las personas

\section{Rossana Vitelli}


Un estudio de Género con Enfoque Territorial: La participación femenina en pequeñas comunidades rurales de Brasil y Uruguay

GRAFICO 1- PARTICIPACION EN ALGUN GRUPO U ORGANIZACIÓN

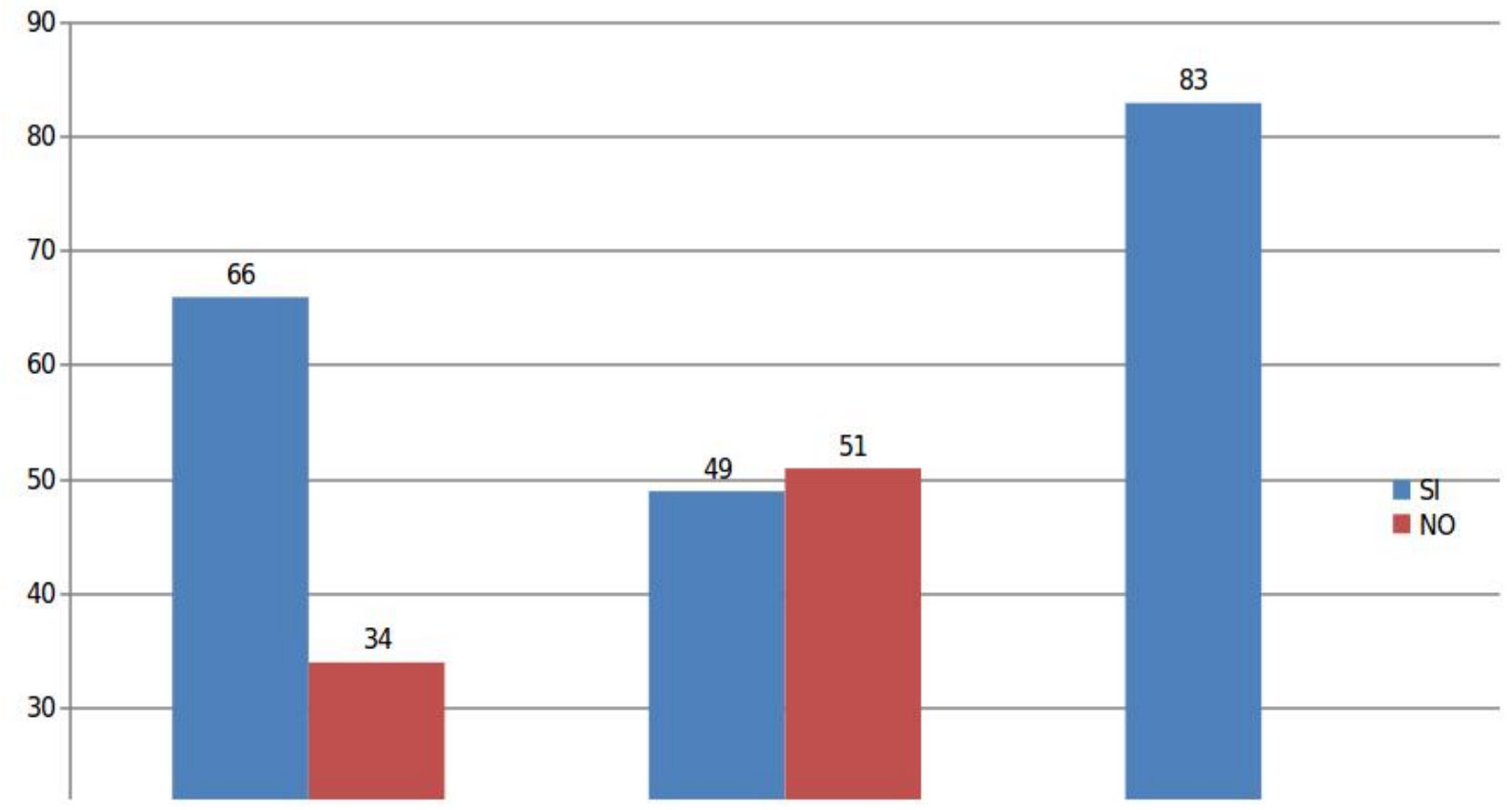

Fuente: Encuesta Participación y ciudadanía en comunidades rurales 2009

GRAFICO 2- PARTICIPACION EN GRUPO U ORGANIZACIÓN. SEGÚN SEXO

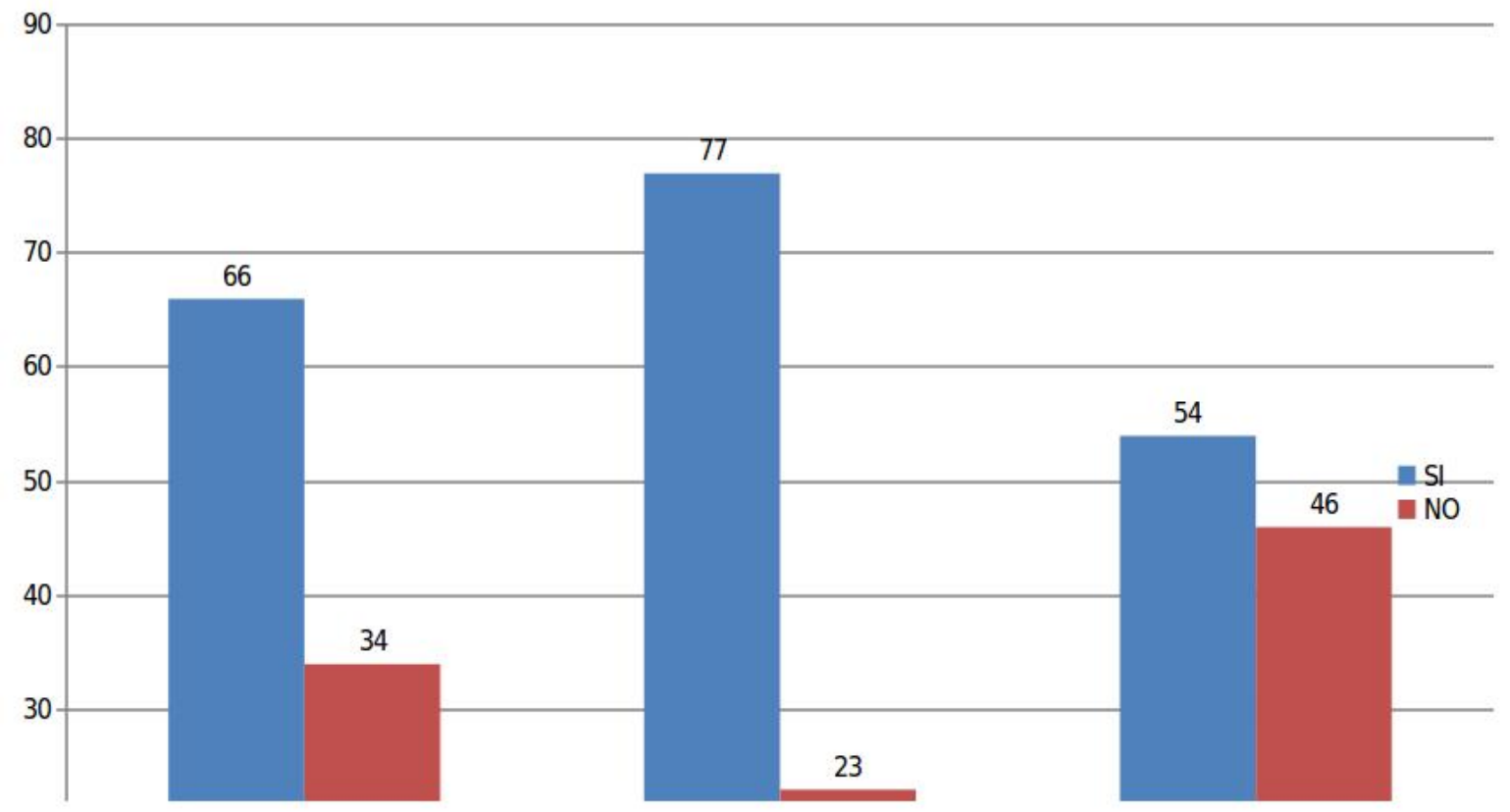

Fuente: Encuesta Participación y ciudadanía en comunidades rurales 2009

Rossana Vitelli 
encuestadas quienes tienen grupo u organización de pertenencia. En definitiva se puede concluir que ambas comunidades se caracterizan por un alto grado de 'asociatividad', siendo que en San Pedro esta característica es un rasgo casi de identidad.

Analizada esta pregunta según el sexo (Grafico 2), aparece una diferencia que marca una mayor participación masculina. En este renglón se ubica el $77 \%$ de los hombres, en tanto representa el 54\% de las mujeres. Este dato preliminar es consistente con situaciones ya largamente estudiadas y que pueden tener dos lecturas o formas de interpretarse: en primer lugar se podría afirmar que todavía existe un mundo 'exterior' y público mayoritariamente ocupado por hombres dejando relegadas a las mujeres al espacio doméstico y privado. Pero, por otro lado, también se puede alegar que más de la mitad de las mujeres encuestadas efectivamente interviene de alguna forma $\mathrm{u}$ otra participando en un grupo u organización, además de las ocupaciones domésticas. Es posible afirmar que esto significa un peso importante de la población femenina incidiendo en la comunidad.

Para profundizar sobre este tema, se indagó sobre el tipo de organizaciones al que pertenecen los encuestados. Se solicitó que indicaran todas aquellas instituciones de las que formaban parte de un listado dado.

Para facilitar el análisis de la tabla 1, podríamos

TABLA 1- TIPO DE ORGANIZACIÓN EN LA QUE PARTICIPA POR COMUNIDAD Y POR SEXO

\begin{tabular}{|c|c|c|c|c|c|}
\hline & $\begin{array}{c}\text { TOTAL } \\
\%\end{array}$ & $\begin{array}{l}\text { SANTA } \\
\text { CRUZ \% }\end{array}$ & $\begin{array}{c}\text { SAN } \\
\text { PEDRO \% }\end{array}$ & $\begin{array}{c}\text { HOMBRES } \\
\%\end{array}$ & $\begin{array}{c}\text { MUJERES } \\
\%\end{array}$ \\
\hline $\begin{array}{l}\text { Parroquia o } \\
\text { Iglesia }\end{array}$ & $32 \%$ & $31 \%$ & $13 \%$ & $38 \%$ & $65 \%$ \\
\hline De vecinos & $26 \%$ & $18 \%$ & $15 \%$ & $44 \%$ & $35 \%$ \\
\hline Escuela & $22 \%$ & $10 \%$ & $15 \%$ & $26 \%$ & $44 \%$ \\
\hline $\begin{array}{l}\text { Club } \\
\text { Deportivo }\end{array}$ & $18 \%$ & $6 \%$ & $14 \%$ & $32 \%$ & $22 \%$ \\
\hline Folklórico & $17 \%$ & $15 \%$ & $8 \%$ & $20 \%$ & $35 \%$ \\
\hline Cooperativa & $8 \%$ & $4 \%$ & $5 \%$ & $15 \%$ & $9 \%$ \\
\hline $\begin{array}{l}\text { Solidaridad o } \\
\text { apoyo a una } \\
\text { causa }\end{array}$ & $7 \%$ & $0 \%$ & $7 \%$ & $12 \%$ & $9 \%$ \\
\hline $\begin{array}{l}\text { Asociación } \\
\text { de Padres }\end{array}$ & $6 \%$ & $2 \%$ & $4 \%$ & $9 \%$ & $9 \%$ \\
\hline Sindicato & $5 \%$ & $8 \%$ & $0 \%$ & $9 \%$ & $4 \%$ \\
\hline $\begin{array}{l}\text { Social, } \\
\text { beneficencia, } \\
\text { o filantrópico }\end{array}$ & $4 \%$ & $0 \%$ & $4 \%$ & $9 \%$ & $4 \%$ \\
\hline $\begin{array}{l}\text { Organización } \\
\text { Politica }\end{array}$ & $3 \%$ & $0 \%$ & $3 \%$ & $3 \%$ & $9 \%$ \\
\hline Gremio & $2 \%$ & $0 \%$ & $2 \%$ & $3 \%$ & $4 \%$ \\
\hline
\end{tabular}

Fuente: Encuesta Participación y ciudadanía en comunidades rurales 2009 
realizar una primera división en el listado de entidades marcadas que corresponde a un quiebre o escalón en el número de adherentes. Este primer grupo de organizaciones concentra los mayores porcentajes de integrantes, y lo conforman la Iglesia, los grupos de vecinos, la escuela, el club deportivo y los grupos folklóricos. La iglesia o la Parroquia aparecen como la institución que alcanza mayor grado de participación de los vecinos, con la advertencia que se incluyó toda y cualquier forma de participación y práctica religiosa. Si examinamos por comunidad, Santa Cruz do Sul tiene un registro mucho mayor que San Pedro de personas que forman parte de una Iglesia, lo que sin duda significa un peso importante de la religión para explicar otros aspectos de la comunidad. Se destaca que la participación en la Iglesia es marcadamente femenina con una distancia importante sobre los hombres. Las otras tres organizaciones que nuclean básicamente la participación de los entrevistados son los grupos de vecinos -aquellos que se forman por cuestiones específicas de vecindad o cercanía- luego la escuela -algo que en las comunidades rurales tiene mucha significación por toda la actividad que se desarrolla en ella-; y finalmente los clubes deportivos y los grupos folklóricos. En este caso, las mujeres participan más que los hombres en la escuela y grupos de folklore, mientras que los hombres están más vinculados a los grupos de vecinos y los clubes deportivos.

En un segundo grupo que ya nuclean bastante menor número de integrantes se ubican las organizaciones que podríamos calificar como de 'ideas' u objetivos claramente 'militantes'. Aquí están las agremiaciones, sindicatos de trabajadores, grupos de solidaridad, diferentes agrupaciones pertenecientes a la Iglesia y organizaciones político-gremiales. Una primera lectura indica que existe una participación superior de los hombres en este tipo de organizaciones en relación a las mujeres, a excepción del caso particular de 'organización política' donde la presencia femenina es mayor. En este núcleo de organizaciones aparece un matiz importante entre comunidades: mientras que se encuentran integrantes de sindicatos en Santa Cruz do Sul, no aparecen respuestas en este renglón en San Pedro que sí tiene participantes de gremios. Esto parece consistente con las características de ambas localidades, dado que en esta última la producción agropecuaria y los negocios de turismo rural son típicamente familiares, lo que induce más bien a una participación en gremios que en sindicatos de asalariados.

Las personas que dentro de sus grupos o asociaciones asumen algún tipo de responsabilidad que implique representación o dirección de los mismos, están a su vez asumiendo un grado mayor de compromiso hacia una causa, objetivo, o finalidad. Es decir, se puede afirmar que ser delegado o directivo de algún colectivo implica involucrarse en forma más comprometida con la comunidad. En este sentido a los encuestados que respondieron tener participación en cualquiera de las organizaciones antes listadas, se les consultó si además ocupaban algún cargo como delegado o representante.

Un $37 \%$ de quienes tienen alguna forma de participación, es también representante, delegado o jerarca. Esto significa un porcentaje bastante importante, y si analizamos el caso de San Pedro es significativo dado que representa un número próximo a la mitad de los encuestados. Si se tiene en cuenta que estamos hablando de un porcentaje importante de un grupo de vecinos que declaró participar y que a su vez era muy alto, se puede concluir en términos generales que el nivel de compromiso con la comunidad bastante fuerte, siendo el caso de San Pedro especialmente significativo esta característica.

Observados estos resultados según sexo, se destaca claramente algo que es consistente con los datos que brindan de la mayoría de los estudios sobre el tema: es una mayoría amplia de hombres quienes ocupan la representación y conducción de las organizaciones, marcando una brecha de género ya que esta representación no corresponde con la participación en

TABLA 2- PARTICIPACION COMO DELEGADO, REPRESENTANTE O JERARCA DE ALGUNA ORGANIZACIÓN

\begin{tabular}{|c|c|c|c|c|c|c|c|c|c|c|c|}
\hline & TOTAL & $\%$ & $\begin{array}{c}\text { Sta. } \\
\text { CRUZ }\end{array}$ & $\%$ & $\begin{array}{c}\text { SAN } \\
\text { PEDRO }\end{array}$ & $\%$ & H & $\%$ & M & $\%$ \\
\hline SI & 21 & $37 \%$ & 5 & $23 \%$ & 16 & $46 \%$ & 16 & $47 \%$ & 5 & $22 \%$ \\
\hline No & 36 & $63 \%$ & 17 & $77 \%$ & 19 & $54 \%$ & 18 & $53 \%$ & 18 & $78 \%$ \\
\hline
\end{tabular}

Fuente: Encuesta Participación y cludadania en comunidades rurales 2009 
la base. Como se encuentra con frecuencia, si bien las mujeres integran los grupos, o bien son relegadas o ellas mismas se autoexcluyen de la representación y conducción de los mismos, punto que se examinará más adelante.

La intensidad de la participación en las comunidades fue medida a partir de la carga horaria semanal destinada por los entrevistados a sus grupos $\mathrm{u}$ organizaciones. Para esto se solicitó a quienes declararon ser integrantes de algún grupo $u$ organización indicar la cantidad de horas que destinaban a esa actividad.

En la tabla 3 se desprende que la mayoría de quienes tienen una actividad de este tipo, le dedican una carga de hasta 4 horas semanales, que podemos considerar 'moderada', representando el $68 \%$ de quienes declaran integrar grupos. Mientras tanto no es menor el hecho de que un $21 \%$ de los encuestados declaran destinar entre 5 y 10 horas semanales, una carga considerada 'media', así como que un $11 \%$ le dedica más de 10 horas por semana, o sea una carga 'alta'.

TABLA 3 INTENSIDAD DE LA PARTICIPACION EN GRUPOS Y ORGANIZACIONES POR COMUNIDAD Y SEXO

\begin{tabular}{|c|c|c|c|c|c|c|c|c|}
\hline & TOTAL & $\%$ & $\begin{array}{c}\text { Sta. } \\
\text { CRUZ }\end{array}$ & $\%$ & $\begin{array}{l}\text { SAN } \\
\text { PEDRO }\end{array}$ & $\%$ & $\mathrm{H} \%$ & $\mathrm{M} \%$ \\
\hline $\begin{array}{c}\text { Hasta } 4 \\
\text { horas por } \\
\text { semana }\end{array}$ & 39 & $68 \%$ & 16 & $73 \%$ & 23 & $66 \%$ & $68 \%$ & $69 \%$ \\
\hline $\begin{array}{l}\text { Entre } 5 \text { y } \\
10 \text { horas } \\
\text { Semana }\end{array}$ & 12 & $21 \%$ & 5 & $23 \%$ & 7 & $20 \%$ & $20 \%$ & $22 \%$ \\
\hline $\begin{array}{l}\text { Más de } \\
10 \text { horas } \\
\text { por } \\
\text { semana }\end{array}$ & 6 & $11 \%$ & 1 & $4 \%$ & 5 & $14 \%$ & $12 \%$ & $9 \%$ \\
\hline TOTAL & 57 & $100 \%$ & 22 & $100 \%$ & 35 & $100 \%$ & $100 \%$ & $100 \%$ \\
\hline
\end{tabular}

Fuente: Encuesta Participación y ciudadanía en comunidades rurales 2009

Si observamos las comunidades, podemos apreciar que en Santa Cruz do Sul se registran más personas con lo que llamamos una tasa moderada de participación, mientras que en San Pedro el porcentaje de quienes dedican más de 10 horas semanales a esta actividad son más del triple que los de la primera comunidad.

Observada la intensidad de la participación de acuerdo al sexo, surge que en los dos primeros tramos la misma es muy similar entre hombres y mujeres. Sólo se advierte una distancia no muy importante en el tramo de la participación alta que registra más integrantes masculinos.

En la siguiente pregunta se intentó registrar la opinión de los entrevistados sobre la participación de acuerdo al sexo, consultando cuál era mayoritaria (Tabla 4).

Existe una diferencia importante en la percepción que hombres y mujeres tienen sobre el papel que ocupan en grupos y organizaciones. Mientras que el $51 \%$ de las mujeres opinan que los hombres participan menos que ellas, un $59 \%$ de los hombres manifiestan que participan igual. El no reconocimiento de una eventual mayor participación femenina refleja la invisibilidad que muchas veces impera en las comunidades de las tareas que efectivamente sí realizan las mujeres, como por ejemplo la registrada a nivel de las escuelas y organizaciones de asistencia religiosas. Sin duda uno de los mecanismos que produce esta poca visualización de las tareas femeninas en la comunidad es la poca valoración social que se tiene sobre algunas de las organizaciones que son -precisamente- las 'piloteadas' por mujeres. En tanto aquellos grupos u organizaciones con mayor presencia masculina, parecen tener mayor apreciación entre los vecinos.

En la siguiente pregunta se intentó indagar acerca de los conceptos más difundidos sobre el comportamiento de las mujeres cuando actúan en reuniones y cuando participan en grupos. Con frecuencia existen juicios y valoraciones que se reproducen rápidamente entre los vecinos sobre la actuación femenina y que -pudiendo estar más cercanas o no a la realidad- quedan instalados en los

Rossana Vitelli 
TABLA 4 - PERCEPCION SOBRE LA PARTICIPACION DE HOMBRES Y MUJERES SEGÚN SEXO

\begin{tabular}{|c|c|c|c|c|c|c|}
\hline & TOTAL & $\%$ & HOMBRES & $\%$ & MUJERES & $\%$ \\
\hline $\begin{array}{c}\text { Participan } \\
\text { igual }\end{array}$ & 40 & $46 \%$ & 26 & $59 \%$ & 14 & $33 \%$ \\
\hline $\begin{array}{c}\text { Los hombres } \\
\text { Participan } \\
\text { menos }\end{array}$ & 32 & $37 \%$ & 10 & $23 \%$ & 22 & $51 \%$ \\
\hline $\begin{array}{c}\text { Las mujeres } \\
\text { participan } \\
\text { menos }\end{array}$ & 15 & $17 \%$ & 8 & $18 \%$ & 7 & $16 \%$ \\
\hline TOTAL & 87 & $100 \%$ & 44 & $100 \%$ & 43 & $100 \%$ \\
\hline
\end{tabular}

\section{Fuente: Encuesta Participación y ciudadania en comunidades rurales 2009}

grupos. Se intercalaron juicios variados, algunos más positivos con otros que no lo son tanto, buscando rastrear algunos estereotipos acerca de este tema. Se solicitó marcar hasta tres opciones de acuerdo con las afirmaciones brindadas y se ubicaron en el orden que se presenta a continuación.

En primer lugar, en la tabla 5 , el $26 \%$ de los encuestados opina que las mujeres dan mayor participación y son más dispuestas y abiertas para el diálogo. En un nivel inferior se agruparon con similar porcentaje juicios igualmente positivos, donde se expresa que aunque hablen menos aportan experiencias valiosas, que trabajan a la par que el hombre y que son menos autoritarias. Con un registro un poco menor se ubica la afirmación de que trabajan más que el hombre. Finalmente y con porcentajes mucho menores aparecen ubicadas las opciones menos favorables, como las que afirman que las mujeres monopolizan la palabra, son más distraídas y desorganizadas o más inseguras. Sin duda las apreciaciones de los entrevistados reflejan que tienen una muy alta valoración acerca de la participación femenina. Examinadas ambas comunidades, en Santa Cruz do Sul aparecen algunas de las afirmaciones positivas sobre el comportamiento de las mujeres con mayor registro que en San Pedro, tal como la afirmación de que las mujeres son menos autoritarias y también la que expresa que trabajan más que el hombre. Sin embargo también en esta comunidad aparece con un registro que triplica el porcentaje encontrado en San Pedro sobre la opinión de que monopolizan la palabra y no dicen cosas importantes en las reuniones con un 7\%.

En principio las dos comunidades manejan una buena opinión sobre el comportamiento de las mujeres en los grupos, sin embargo sumadas las respuestas más negativas de los últimos tres renglones, éstas representan un $12 \%$ de las opciones en Santa Cruz do Sul y un $7 \%$ en San Pedro, lo que no deja de representar cierto impacto en el conjunto. Hay que tener presente que en Santa Cruz se encontró mayor militancia feminista y conciencia de género, lo que puede provocar este efecto 'rechazo' por parte del conjunto de la población cuando se pregunta por los estilos de participación femenina.

\section{Algunas Reflexiones Finales}

A través de la investigación realizada se ha podido verificar que tanto en San Pedro como en Santa Cruz do Sul existe un número importante de grupos y organizaciones, con una alta tasa de participación de los vecinos. Estas agrupaciones son muy diversas, y fueron catalogadas como 'de base y socialización' por un lado, y de 'ideas o de militantes' por otro. En este sentido, las mujeres tienen un nivel de participación tan importante como el de los hombres. Acá se han encontrado diferencias entre ambas comunidades. La historia, la tradición y la cultura en Santa Cruz do Sul, Brasil, parece haber conformado una política más abierta a la participación de mujeres en organizaciones del tipo que llamamos de 'ideas o militantes"'

\section{Rossana Vitelli}


Un estudio de Género con Enfoque Territorial: La

participación femenina en pequeñas comunidades

rurales de Brasil y Uruguay

TABLA 5- VALORACIÓN SOBRE EL COMPORTAMIENTO DE LAS MUJERES EN LOS GRUPOS Y ORGANIZACIONES

\begin{tabular}{|c|c|c|c|c|c|c|}
\hline & TOTAL & $\%$ & $\begin{array}{l}\text { SANTA } \\
\text { CRUZ }\end{array}$ & $\%$ & $\begin{array}{l}\text { SAN } \\
\text { PEDRO }\end{array}$ & $\%$ \\
\hline $\begin{array}{l}\text { Dan más participación a los } \\
\text { demás y son más abiertas y } \\
\text { dispuestas al diálogo }\end{array}$ & 57 & $26 \%$ & 29 & $24 \%$ & 28 & $28 \%$ \\
\hline $\begin{array}{l}\text { Hablan menos que los hombres } \\
\text { pero aportan experiencias } \\
\text { valiosas }\end{array}$ & 38 & $17 \%$ & 21 & $17 \%$ & 17 & $17 \%$ \\
\hline Trabajan a la par del hombre & 38 & $17 \%$ & 11 & $9 \%$ & 27 & $27 \%$ \\
\hline $\begin{array}{l}\text { Son menos autoritarias que los } \\
\text { hombres y más democráticas }\end{array}$ & 35 & $16 \%$ & 26 & $21 \%$ & 9 & $9 \%$ \\
\hline Trabajan más que el hombre & 30 & $14 \%$ & 19 & $16 \%$ & 11 & $11 \%$ \\
\hline $\begin{array}{l}\text { Monopolizan la palabra en las } \\
\text { reuniones y no dicen cosas } \\
\text { importantes }\end{array}$ & 10 & $5 \%$ & 8 & $7 \%$ & 2 & $2 \%$ \\
\hline $\begin{array}{l}\text { Son más conversadoras y } \\
\text { distraídas y por eso resultan } \\
\text { menos organizadas y eficientes }\end{array}$ & 6 & $3 \%$ & 4 & $3 \%$ & 2 & $2 \%$ \\
\hline $\begin{array}{l}\text { Son más inseguras y a veces } \\
\text { dificultan la tarea }\end{array}$ & 6 & $3 \%$ & 3 & $2 \%$ & 3 & $3 \%$ \\
\hline
\end{tabular}

Fuente: Encuesta Participación y ciudadanía en comunídades rurales 2009

(sindicatos, cooperativas, grupos políticos) mientras que en San Pedro, Uruguay, las mujeres no han encontrado espacios en este tipo de asociaciones, concentrándose en las organizaciones que nombramos como 'de base' (escuelas, grupos de vecinos, grupos folklóricos, clubes).

Se han encontrado algunas diferencias importantes en la forma que adopta la participación femenina en ambas comunidades. Esto, según se pudo extraer de las entrevistas y visitas realizadas, responde a los mecanismos por los cuales se fueron construyendo -tanto histórica como culturalmente- las concepciones y los valores acerca de cuáles deben ser los roles a cumplir tanto por hombres como por mujeres en sus comunidades. En este sentido tanto las políticas específicas dirigidas a la promoción de la concientización de género -que difieren de un país a otro- como algunos elementos tradicionales de la cultura: como ser la religión, el origen de las migraciones que dieron origen a cada comunidad, como también las propias prácticas locales, han determinado que se hayan encontrado concepciones más abiertas y una participación más 'concientizada' en términos de género en Santa Cruz do Sul, mientras que la integración de las mujeres en las organizaciones y grupos en San Pedro responde a los valores, una cultura y una historia más tradicionales.

En el caso de San Pedro, encontramos una comunidad rural con organizaciones tradicionales que reproducen estereotipos y prácticas de inequidad en sus concepciones de género. En tanto Santa Cruz do Sul se manifestó como una comunidad donde existen organizaciones que producen prácticas de socialización de base y donde tal vez se reproduzcan valores y prácticas tradicionales de género, pero también aparece otro conjunto de organizaciones y grupos que promueven prácticas que hacen a un proceso más comprometido con ciertas causas que promueven cambios. Estas prácticas están montadas sobre unos pilares compuestos por fundamentos ideológicos, religiosos y normativos. Entre estos fundamentos también se encuentran las concepciones de género.

Se podría señalar que ciertamente la participación masculina también genera ciudadanía, siendo posible que ellos intervengan en su difusión y reproducción. Sin embargo este último rasgo no es la característica o

Rossana Vitelli 
función esencial de esa intervención. Lo que pudo ser evidenciado es que las formas de participación masculina apuntan a generar formas más verticales o 'de escalera' en la producción de capital social. Entonces, se puede afirmar que la especialización de la participación masculina suele darse en organizaciones que manejan temas involucrados con el poder, y que a su vez articulan en forma de puente con otras instituciones que detentan poder $y$ dan más posibilidades de visibilidad y proyección, mientras que la especialización femenina de la participación suele adoptar formas más horizontales y destinadas a la trasmisión y reproducción de los valores y prácticas ciudadanas locales.

Traducción propia del original en Portugués

\section{Referências}

ABRAMOVAY, Ricardo. O capital social dos territórios: repensando o desenvolvimento rural. Economia Aplicada, v. 4, n. 2, p. 379 - 397, abril/junho 2000.

AGUIRRE, Rosario. Género, ciudadanía social y trabajo. Facultad de Ciencias Sociales. Doble Montevideo: Clic Editoras., 2003.

ARRIAGADA, Irma. Capital social: potencialidades y limitaciones analíticas de un concepto. Estudios Sociológicos, v. XXI, n. 3, p. 557-584, 2003.

BANUETT, Marcelle. Perspectiva de Género en la nueva ruralidad. IICA/ASDI, 1999.

BOURDIEU, Pierre. La dominación masculina. Editorial Anagrama: Barcelona, 2000.

. Le capital social. En Actes de la recherche en sciences sociales, v. 31, n. 1, p. $2-3,1980$.

BOURDIEU, Pierre; WACQUANT, Loïc. Respuestas por una antropología reflexiva. México: Ed. Grijalbo, 1995.

CAMPAÑA, Pilar. El contenido de género en la investigación en sistemas de producción. Santiago de Chile: Ed. Interamericana Ltda,1992.

CANABAL, Beatriz. La mujer campesina como sujeto de investigación. Formas de investigación y acción.
Revista Mexicana de Sociología, v. 56, n. 2, p. 89 103, 1994.

CÔRTES VARGAS, Soraya. Capital social, redução de desigualdades e sinergia das esferas pública e privada In: XXV Encontro Anual da ANPOCS, Caxambu, 2001 .

COUTINHO, Carlos. Cidadania e modernidade. Perspectivas, v. 22, p. $41-59,1999$.

DE LEÓN, Kirai. El Uruguay Rural y sus Mujeres: producción, trabajo y organización. GRECMU: Montevideo: GRECMU, 1993.

DEERE, Carmen; LEÓN, Magdalena. La brecha de género en la propiedad de la tierra en América Latina. In: Seminario EL MUNDO RURAL: TRANSFORMACIONES Y PERSPECTIVAS A LA LUZ DE LA NUEVA RURALIDAD“ Bogotá, Universidad Javeriana, 15 al 17 de octubre de 2003.

Planteamientos teóricos y metodológicos para el estudio de la mujer rural y el proceso de desarrollo del capitalismo. Em: DEERE, Carmen Diana, Magdalena León; REY, Nohra (Eds). Mujer y Capitalismo Agrario. Bogotá: Asociación Colombiana para el Estudio de la Población, 1980, capítulo 1 .

DURSTON, John. El capital social campesino en la gestión del desarrollo rural. Díadas, equipos, puentes y escaleras. Santiago: CEPAL, 2002.

FRASER, Nancy. Da redistribuição ao reconhecimento? Dilemas da justiça na era póssocialista. In: SOUZA, Jessé (org.). Democracia hoje; novos desafios para a teoria democrática contemporânea. Brasília: UNB, 2001, p. 245 - 282.

GARCÍA, Cristóbal H. Ciudadanía, Capital Social y Solidaridad. (Mimeo). ISUC, Chile, 2001.

GEHLEN, Ivaldo; RIELLA, Alberto. Dinâmicas territoriais e desenvolvimento sustentável. Revista Sociologías, v. 6, n. 11, p. 20 - 26, 2004.

GIDDENS, Anthony. La transformación de la intimidad. Sexualidad, amor y erotismo enn las sociedades modernas. Madrid: Ed. Cátedra, 1995.

GRANOVETTER, Mark. Economic Action and Social Structure: the Problem of Embeddedness. American Journal of Sociology, v. 91, n. 3, p. 481 - 510, 1985. 
LECHNER, Norbert. Desafíos de un desarrollo humano: individualización y capital social. En KLIKSBERG, Bernardo; TOMASSINI, Luciano (comps.). Capital social y cultura: claves estratégicas para el desarrollo. Buenos Aires, BID / Fund. Felipe Herrera / Univ. Maryland / FCE, 2000 (original mimeo, Paris, 1999).

MARSHALL,T. H.; BOTTOMORE, Tom. Ciudadanía y clase social. Madrid: Alianza Editorial, 1998.

MEILlASSOUX, Claude. Mujeres, graneros y capitales. España: Siglo XXI editores, 1978.

MONTAÑO, Sonia. Políticas para el empoderamiento de las mujeres como estrategia de lucha contra la pobreza. Em: ATRIA, R. et al. (Comp.), Capital Social y reducción de la pobreza en América Latina y el Caribe: en busca de un nuevo paradigma. Santiago de Chile: CEPAL / Michigan State University, 2003, p. $361-378$

PINTO, Céli Regina Jardim. Movimentos sociais: espaços privilegiados da mulher enquanto sujeito político. In: COSTA, Albertina de O.; BRUSCHINI, Cristina (Orgs.). Uma questão de gênero. Rio de Janeiro: Rosa dos Tempos / Fundação Carlos Chagas, 1992, p. 127-150.

Participação (Representação?) Política da Mulher No Brasil: Limites e Perspectivas. In: SAFFIOTI, Heleieth; VARGAS, Monica Muños. (Org.). Mulher Brasileria é assim. Rio de Janeiro: Rosa dos Ventos, 1994, p. 195-230.

PIÑEIRO, Diego. Repensando la ruralidad: población $\mathrm{y}$ trabajadores rurales en el contexto de transformaciones agrarias. Ponencia presentada al XXII CONGRESO DE ALAS, Chile, 1999.

PUTNAM, Robert. Making democracy work. Civic traditions in Modern Italy. United Kingdom: Princeton University Press, 1994.

REBOLLEDO, Loreto. Las mujeres rurales en el contexto de la modernización agraria. Revista Anales, Sexta serie, n. 5, p. 99-133, 1997.

RIELLA, Alberto, VITELLI, Rossana. Escuelas rurales y desarrollo territorial: una reflexión para el caso uruguayo. Revista Pampa, n.1, p. 131 - 146, 2005.

SCHNEIDER, Sérgio. Agricultura familiar e industrialização: pluriactividade e descentralização industrial no Rio Grande do Sul. Porto Alegre: Editora Universidade - UFRGS, 1999.

VARELA, Julia; ÁlVAREZ-URÍA, Fernando. Sociología del Género. Algunos modelos de análisis. Archipiélago - Cuadernos de crítica de la cultura, n. 30, p. $11-21,1997$.

VITELLI, Rossana. Capital social, participación y ciudadanía en el medio rural. Una perspectiva de género. 2010. Tesis de doctorado - Instituto de Filosofia e Ciências Humanas - Programa de PósGraduação em Sociologia . Porto Alegre.

La situación de las mujeres rurales en Uruguay. Santiago de Chile: FAO - Oficina Regional para América Latina y el Caribe, 2005.

WILSON, Fiona. La mujer y las transformaciones agrarias en América Latina: revisión de algunos conceptos que fundamentan la investigación. En: SPINDEL, Cheywa R.; LEÓN, Magdalena; DEERE, Carmen Diana (Eds). La mujer y la Política Agraria en América Latina. Colombia: Siglo XXI Editores, 1986, p. $265-290$. 